Published in Surf. Sci. 606, 28 (2012).

\title{
Structure and Electronic Spectroscopy of Steps on GaAs(110) Surfaces
}

S. Gaan and R. M. Feenstra

Dept. Physics, Carnegie Mellon University, Pittsburgh, PA 15213, USA

Ph. Ebert and R. E. Dunin-Borkowski

Peter Grünberg Institut, Forschungszentrum Jülich GmbH, 52425 Jülich, Germany

J. Walker and E. Towe

Dept. Electrical and Computer Engineering, Carnegie Mellon University, Pittsburgh, PA 15213, USA

\begin{abstract}
Steps on GaAs(110) surfaces, with step-normal vectors parallel to [001], are studied by scanning tunneling microscopy and spectroscopy. Two possible orientations of the steps occur, with outward normal vectors of [001] or [00 1 ], which in simple bulk-terminated form have Ga (cations) or As (anions) on their edges, respectively. The latter type of step in n-type or undoped material is found to retain its bulk-terminated form. A band of states is observed extending out from the valence band, associated with the dangling bonds of the terminating As atoms. It is argued that compensation of the dangling bonds on the step edges is the driving force for the step structure, producing reconstruction of the step edges in certain cases.
\end{abstract}




\section{Introduction}

Steps on semiconductor surfaces are well known to play an influential role in both surface chemical reactions and epitaxial growth. ${ }^{1}$ These processes are impacted by the electronic structure of step edges, which can lead to states in the band gap and hence charging of the step edges. The (110) surface of GaAs and other III-V semiconductors have been previously demonstrated to display charging of their step edges. ${ }^{2,3,4}$ The sign of the charge was found to depend on the doping of the semiconductor, but a detailed understanding in terms of the geometric and electronic structure of the step edges was lacking. From a theoretical perspective, steps of various orientations on GaAs(110) have been studied. ${ }^{5,6}$ For perhaps the simplest step orientation with a normal vector along [001], a prior study has predicted an electronic structure of the step edges that does not differ substantially (i.e. without states in the gap) from the bulk band structure. ${ }^{6}$ However, other studies point out the intrinsic instability of such steps, since it is difficult to achieve charge neutrality (auto-compensation) of the step edges. ${ }^{5}$ Additional experimental work is needed to address this issue, e.g., by fully determining the geometric and electronic structure of the step edges.

In this work we examine steps on GaAs(110) surfaces using scanning tunneling microscopy and spectroscopy (STM/S). We focus on steps that run along the [1 10$]$ directions, i.e. with normal vector along [001]. Two types of such steps exist as pictured in Fig. 1; in a bulk-terminated form one step has As dangling bonds (DBs) and the other has Ga DBs. Prior to any electron transfer, each As DB bond is formally occupied by $5 / 4$ of an electron and each Ga DB by 3/4 of an electron. This situation with partial occupation is energetically unfavorable as mentioned above, and so, if possible, electron transfer (e.g. via doping) occurs such that the As (anion) DBs become completely filled and/or the Ga (cation) DBs become completely empty. We find that this type of electron transfer does indeed occur for n-type or p-type material, respectively. Other situations, with anion-edge steps in p-type material or cation-edge steps in n-type material lead to reconstructions of the step edges.

\section{Experimental Results}

Clean $\operatorname{GaAs}(110)$ surfaces were obtained by cleaving in ultra-high-vacuum at pressure $<1 \times 10^{-8} \mathrm{~Pa}$. Both commercial Pt-Ir tips as well as electrochemically etched $\mathrm{W}$ tips were used, with in situ cleaning performed by electron bombardment or by annealing. ${ }^{2,9}$ STM images are acquired with constant current values and sample-tip voltages as specified. Conductance spectra are measured and normalized in the same manner as previously described, ${ }^{9}$ using a lock-in amplifier and a modulation voltage of $50 \mathrm{mV}$. Spectra are acquired simultaneously with image acquisition ${ }^{9}$ so that the uncertainty in the precise location of each spectrum is less than $0.5 \mathrm{~nm}$. All measurements were performed at room temperature.

Figure 2 shows STM images obtained across both As-edge and Ga-edge steps on Sidoped $\left(1.3 \times 10^{19} \mathrm{~cm}^{-3}\right)$ n-type GaAs(110). All steps correspond to a single monoatomic step with a height of $0.20 \mathrm{~nm}$. The orientation of the step edges is known from voltage- 
dependent STM imaging of the As and Ga atoms on the surface. Below each image in Fig. 2 are shown constant-current contours across the locations indicated in the images. For the As-edge steps on n-type material, Fig. 1(a), the constant-current contour displays an increased height across the step edge for negative sample voltages, and a decreased height for positive voltages. This behavior demonstrates that the As-edge step is negatively charged. ${ }^{7}$

The Ga-edge step of Fig. 2 displays more complicated behavior, with a predominantly $2 \times$ reconstruction along the step edge. However, like the As-edge step, the constantcurrent contours for the Ga-edge step display a slightly increased height across the step edges for negative sample voltages, and a decreased height for positive voltages, indicative of negative charge at the step edge. But the magnitude of the charge is considerably less than for the As-edge step.

High resolution images of the As-edge step on n-type GaAs(110) are displayed in Fig. 3 . We find a clear $1 \times$ periodicity (period $0.40 \mathrm{~nm}$ ) along the step. This behavior is consistent with a simple bulk-terminated model for the step edge, as shown in Fig. 1(a). We therefore tentatively identify the structure as being the same as in Fig. 1(a), and spectroscopic results described below support this identification. We find that the states associated with the As DBs are located within the valence band (VB) and/or extending slightly out of the VB. For n-type material, doping electrons can transfer from the conduction band $(\mathrm{CB})$ edge to the dangling band states, thereby reducing the energy of the system and stabilizing this structure.

In contrast, the Ga-edge steps clearly have a structure that is different from that of Fig. 1(b). In this case, assuming the energy of these states to be located in the upper part of the band gap or within the $\mathrm{CB}$, it is not possible to fully empty those dangling bonds through electron transfer because there are no available states in the VB for the present case of ntype doping. However, for p-type material empty VB states are available, and indeed, on p-type InP surfaces we find no apparent reconstruction on the analogous In-edge steps (but those surfaces show a $2 \times$ reconstruction for the P-edge steps). ${ }^{8}$ Thus, we interpret the $2 \times$ reconstruction as providing some other means of lowering the energy of the system.

Studies have also been performed on undoped GaAs. The sample in this case contained layers of InAs quantum dots (QDs), surrounded by GaAs layers. ${ }^{9}$ This heterostructure is viewed in cross-section, along a cleaved (110) face, as shown in Fig. 4(a). The QDs do not influence the structure of the steps, but strain associated with the dots do tend to produce steps on the surface during the cleavage process. Detailed STS measurements were made at various locations, ${ }^{9}$ including near surface steps, as indicated in Fig. 4(a).

A cross-sectional cut across the step seen in Fig. 4(a) is shown in Fig. 4(b). This step, as well as all other [00 $\overline{1}$ ]-oriented (As-edge) steps on the undoped surfaces, exhibit an increased surface height near the step edge for negative sample voltages, consistent with negative charge on the step. This is the same result as found for the n-type surfaces shown in Figs. 2 and 3. In contrast, most Ga-edge steps display a decreased height, with 
STM images of those steps showing typically no observable reconstruction along the step edge. Those observations are consistent with the p-type results mentioned above. We conclude that the results for undoped surfaces are somewhat in between those for n-type and p-type surfaces. But, most importantly for the interpretation of our spectroscopic results, we do find the As-edge steps to have no reconstruction, suggestive of the same As-terminated structure as pictured in Fig. 1(a).

Tunneling spectra acquired from the surface locations indicated in Fig. 4(a) are shown in Fig. 5. At the relatively large distance of $12 \mathrm{~nm}$ from the step edge, spectra $c$ and $f$, we observe spectra typical of the clean GaAs(110) surface: the VB is seen at negative voltages and the $\mathrm{CB}$ at positive voltages, with these bands being separated by an apparent band gap with width of about $1.5 \mathrm{eV}$ (close to the known band gap of GaAs of $1.42 \mathrm{eV}$ ). As the step edge is approached, the spectra are modified due to the presence of the InAs layer containing the QDs, as discussed in detail elsewhere. ${ }^{9}$ The major effect of the QDs is to introduce an electron state in the upper part of the gap. Spectra $b$ and $e$ of Fig. 5 were acquired $4 \mathrm{~nm}$ from the center of the QD layer and about $2 \mathrm{~nm}$ from the step edge. They still display some vestige of the QD electron state. More importantly, the apparent VB onsets have shifted from -0.5 or $-0.4 \mathrm{~V}$ for spectra $c$ and $f$, respectively, to about -0.2 or $-0.1 \mathrm{~V}$ for $b$ and $e$, respectively. Finally, for spectra $a$ and $d$ which are acquired $1 \mathrm{~nm}$ from the step edge, we find a band of states emerging from the $\mathrm{CB}$ and extending throughout the gap. Additionally, the apparent VB onsets in those spectra have further shifted to higher voltages by another $0.1 \mathrm{~V}$.

We thus observe two main spectroscopic features associated with the steps, the shift in the apparent VB onset and the broad band of state extending out from the CB. Regarding the VB edge, it is difficult to distinguish between a shift in the position of the bulk VB onset compared to a set of new states, extending out from the bulk VB, associated with the step. However, examining spectra $a$ and $d$, we see that the observed gap between the apparent VB and CB is only about $1.1 \mathrm{~V}$ wide, much less than the $1.42 \mathrm{eV}$ band gap. In the following Section we quantify this apparent gap by comparing the measured spectra with theoretically computed ones. We demonstrate that the apparent VB onset of the observed spectra is inconsistent with a simple electrostatic shift in the bulk VB edge (i.e. from the charge on the step). Rather, the observed shift arises from new states associated with the step, with these states emerging out from the bulk VB.

We therefore identify the band of states extending out from the VB up to about $0.4 \mathrm{eV}$ above the VB edge (spectra $a$ and $d$ of Fig. 5) as being the As DB states of the Asterminated step. Prior tight-binding computations by Kang et al. did not reveal any such states within the band gap, ${ }^{6}$ but further theoretical modeling employing more sophisticated techniques are probably needed in order to achieve a more reliable comparison between experiment and theory.

Concerning the additional states found at higher energies within the band gap, between about 0.1 and $0.7 \mathrm{eV}$ in Fig. 5, these are clearly also associated with the step but they are not consistent with expectation for As DBs. We therefore hypothesize that these states arise from additional compensating defects at the step edge. These defects could be 
adsorbates, or perhaps even vacancies near the step. If these defects are electron donors, then there would be a strong driving force for them to form since by donating their electrons the charge of the step is reduced, hence lowering the electrostatic energy (and total energy) of the system. In Fig. 4(a), we do in fact find some evidence for defects along the step, as shown by the line cut of Fig. 4(c) which is taken along the step edge. We find an irregular corrugation along the step edge, with amplitude of about $0.02 \mathrm{~nm}$. This amplitude is significantly less than what we find, e.g. for $2 \times$ reconstructed Ga-edge steps $(0.07 \mathrm{~nm}$ corrugation amplitude), but it seems reasonable for adsorbates or perhaps vacancies that are located on the lower terrace immediately adjacent to the step edge. There are regions of this line cut that display $2 \times$ or $3 \times$ spacing between features; an example of the latter is marked in Fig. 4(c).

\section{Theoretical Results}

To assist with interpretation of the experimental results, we compute STM constantcurrent contours using a model previously described in which the semiconductor is treated in an effective-mass (envelope function) approximation. ${ }^{10,11}$ The electrostatic potential arising from the presence of the probe-tip and from the surface steps is computed with a finite-element method. The distribution of states associated with steps is specified, and these states are then occupied according to the Fermi-level energy of the semiconductor, as illustrated in Fig. 6. The potential is determined as a solution of Poisson's equation, including the boundary condition at vacuum/semiconductor interface in which the difference in normal component of the electric displacement is equal to the surface charge density at that point.

We initially assume a band of states associated with the step edge, but neglect any additional states from compensating defects. For the example shown in Fig. 6 we assume n-type doping $\left(1 \times 10^{18} \mathrm{~cm}^{-3}\right)$, with a band of step states $0.4 \mathrm{eV}$ wide containing 2 states (one for each spin) per unit cell along the step edge, i.e. 12.5 states $\mathrm{nm}^{-1} \mathrm{eV}^{-1}$. We place the charge neutrality level at the position corresponding to $5 / 4$ electron occupying this band $(0.25 \mathrm{eV}$ above the lower edge of the band), and we assume that the band is located in the lower part of the GaAs band gap, with charge neutrality level at $0.3 \mathrm{eV}$ above the VB maximum. This distribution is intended to model the [00 $\overline{1}]$-oriented, As-edge step, in which the As DBs create the band of states lying near the VB. Spatially, these step states are assumed to be spread out over $2 \mathrm{~nm}$ centered on the step edge, so that the resulting two-dimensional density-of-states (DOS) has magnitude 6.25 states $\mathrm{nm}^{-2} \mathrm{eV}^{-1}$. Electrons from the n-type dopant levels transfer to the band of step states. Consequently the step becomes negatively charged, and this charge creates an upwards going electrostatic potential centered on the step. The energy of the band of step states therefore rises, in accordance with the potential, until the band is high enough so that the Fermilevel intersects it and thusly restricts the charge on the step. Our model also includes the presence of the probe-tip, although as is apparent from Fig. 6(b) (where the probe-tip is centered at the position $+5 \mathrm{~nm}$ ), the effect of the tip is modest compared to that of the charged step itself. 
The parameters in the computation are the tip radius-of-curvature, tip-sample separation, contact potential (difference in work functions of tip and sample), semiconductor parameters (doping, dielectric constant, etc.) as well as the specified distribution(s) of surface states. Given the electrostatic potential thus obtained, together with specification of additional semiconductor parameters such as band gap, effective masses, etc., the tunnel current is computed using a plane-wave expansion method. ${ }^{9}$ This computation of the tunnel current explicitly includes contributions from GaAs bulk-like states (i.e. as modified by the varying electrostatic potential), but does not include contributions from any surface states such as those from the steps. However, since we are computing constant-current contours at voltages of +2 and $-2 \mathrm{~V}$, it appears from the spectra of Fig. 5 that contributions at those voltages occur primarily from the bulk-like bands and not the step states. The results described here were obtained with similar parameters as described in Ref. [9], except that no QDs were included in the present case. ${ }^{12}$ The model includes a surface step located every $50 \mathrm{~nm}$ along the surface, corresponding to the distance between the QD layers, since each QD layer produces one or more steps on the cleavage surface. ${ }^{9}$

We briefly note one special situation that has a small impact on results, namely, when the CB edge moves below the Fermi-level so that accumulation of carriers occurs in the $\mathrm{CB}$ [as can be seen in the right-hand side of Fig. 6(b), for surface locations opposite the apex of the probe-tip]. It has recently been demonstrated that this sort of accumulation in the CB is greatly restricted by the presence of the GaAs(110) X-point surface states, ${ }^{13,14}$ and such states are fully included in our computations.

Within our electrostatic model, the charge on the step is thusly determined, and it turns out to be about 0.25 electrons per unit cell $(0.40 \mathrm{~nm})$ along the step edge for the situation we are considering in Fig. 6. We point out that this value for the charge is only weakly dependent on the effective width of the step edge, $2 \mathrm{~nm}$, which we have assumed in our computation. If a narrower or wider width is assumed, then the charge on the step edge does change, but only by about $20 \%$ of the change in the width. Much more sophisticated computations that truly model the wavefunctions of the step states would be needed to properly determine the true value of the width. In any case, we find below that our computational results are in semi-quantitative agreement with the measured constantcurrent contours, i.e. within about a factor of two, so it appears that our assumed width of $2 \mathrm{~nm}$ is a reasonable value.

Figure 7 shows a compilation of computational results for both n-type and undoped surfaces, and where we are considering two different distributions of the step states, one with the states in the lower part of the bulk band gap (charge neutrality level $0.3 \mathrm{eV}$ above the VB edge, as in Fig. 6) and the other with the states lying within the VB (charge neutrality level $0.3 \mathrm{eV}$ below the $\mathrm{VB}$ edge). We display constant-current contours as the tip scans across the step edge (i.e. scanning in the $[00 \overline{1}]$ direction), together with the computed charge on the step edge when the probe-tip is fixed at a location above the step and charge is plotted as a function of distance along the step edge ([1 $\overline{1} 0]$ direction), relative to the tip location. For n-type doping, Figs. 7(a) and 7(b), the computed constantcurrent contours reveal negatively-charged behavior for the step edges (i.e. the contours 
show an increased surface height for negative sample voltage and a decreased height for positive voltage). The same behavior is seen whether the band of step states is in the gap or within the VB. In both cases, electron transfer occurs between the donor states and the step states, producing negative charge on the steps for any sample-tip voltage. The same situation is found for the undoped surface with the step states within the VB, Fig. 7(d), in which case the electron transfer occurs from states near the top of the VB into the band of step states.

However, the charging behavior of the step is different when the surface is undoped and the band of step states is within the band gap, Fig. 7(c). Then, the constant-current contours reveal increased surface height for both negative and positive sample voltages. Thus the step is found to be negatively charged for negative sample voltage and positively charged for positive sample voltage. This new behavior comes about since there are no occupied states to contribute electrons to the step states. Rather, the charge on the step is produced in response to the band bending induced by the probe-tip. When the tip Fermi-level is below that of the sample (i.e. negative sample bias) then there is downwards tip-induced band bending. For a fixed position of the Fermi-level, this downwards band bending will cause electrons to accumulate at the step edge (the source of the electrons being the step edge itself, with electrons coming from far away over to locations on the step edge near the probe-tip). Conversely, with positive sample bias there is upwards band bending, and fewer electrons are in the step states at locations near the probe-tip.

Returning to the experimental results, for the undoped material at negative sample voltage we observed increased tip height near the As-edged steps, in agreement with both Figs. 7(c) and 7(d). ${ }^{15}$ We have not performed voltage-dependent STM imaging to obtain constant-current contours at positive sample voltage, but we can deduce that information from the measured tunnel current vs. voltage (obtained simultaneously with the conductance spectra of Fig. 5). We compare the ratio of the tunnel current at $-2 \mathrm{~V}$ to that at $+2 \mathrm{~V}$, for tip positions far from the step (spectra $c$ and $f$ ) compared to near the step (spectra $a$ and $d$ ). As seen in Fig. 5, for the conductance, $d I / d V$, we find that these two ratios are nearly equal. Similarly, for the current, we find that this ratio differs by $<5 \%$ between the two tip positions. This small difference demonstrates that a constant-current contour, at positive sample bias, would have the same behavior as for negative sample bias, i.e. increased tip height near the step edge. The experimental results are therefore in agreement with Fig. 7(c), with the band of step states located within the band gap.

Figure 8 shows a comparison of computed spectra with the experimental results, for the same assumed states as in Fig. 7(c). As a reminder, the computations of conductance include only the bulk states of GaAs, with the states associated with the step being included in terms of their electrostatic charge but with no consideration of their contribution to the conductance. In the $\mathrm{CB}$, good agreement is found between experiment and theory far from the step (spectrum $c$ ), and similarly good agreement is found near the step (spectrum $a$ ) so long as we focus on the portion of the spectra above about $1.1 \mathrm{~V}$ where the step states do not dominate. In the VB the agreement between experiment and theory is much worse, and we argue below that this discrepancy arises because of 
transport-related charging of the compensating defects at the step edge. Such an effect produces slower turn-on of the current and conductance in the VB than would otherwise occur, so that the measured conductance is less than the computed one. Nevertheless, the position of the onset of the VB is accurately given in the computations, since at the onset the current goes to zero and the charging effect is thus absent. Far from the step (spectrum $c$ ) we find good agreement between experiment and theory for the VB onset. Near the step (spectrum $a$ ) we find that the apparent VB onset in the experiment extends about $0.3 \mathrm{~V}$ further into the band gap than the computed VB onset. We therefore identify those states extending into the gap as intrinsic surface states derived from the step edge.

At higher energies in the gap, between about 0.1 and $0.7 \mathrm{~V}$ in Figs. 5 or 8 , we identified (in the previous Section) that conductance as arising from compensating defects at the step edge. We believe that those midgap states, in addition to producing the observed midgap conductance, are also responsible for the significant discrepancy between experiment and theory that is found for the VB conductance between about -1.5 and $-0.6 \mathrm{~V}$. For this same voltage range we find that the measured values for the decay constant of the tunnel current, i.e. $\kappa$ where $I \propto \exp (-2 \kappa s)$, are substantially lower than ideal values of $\approx 10 \mathrm{~nm}^{-1}$. Our measured value for $\kappa$ averaged over all voltages is $7.5 \mathrm{~nm}^{-1}$, as previously described. ${ }^{9}$ But, if we separately compute averages over positive and negative voltages, we find $\kappa$ values of about $6 \mathrm{~nm}^{-1}$ for the negative and $9 \mathrm{~nm}^{-1}$ for the positive voltages. The former are very much less than ideal, hence indicating some new phenomenon occurring in the transport.

Nonequilibrium carrier occupation on the surface has been observed in prior STM/S studies, ${ }^{16,17,18,19,20}$ and reduced $\kappa$ values have been associated with this effect. ${ }^{19,20}$ For the present situation, at negative sample voltages, the Fermi-energy at the surface will intersect the states induced by the compensating defects. Transport through these states (e.g. hopping) is likely quite limited. Hence, compared to equilibrium occupation, there will be additional positive charge along the step edge [the model used here is very similar to that previously discussed for defects along surface domain boundaries on $\mathrm{Ge}$ $(111) \mathrm{c} 2 \times 8$ surface]. ${ }^{18}$ This positive change will produce additional downwards band bending, hence reducing the current and producing less-than-ideal $\kappa$ values. We do not attempt more detailed modeling of the spectra, but qualitatively at least, we believe that these effects can account for the discrepancy we find between the theory and experiment at negative voltages.

Finally, we return to consider the effects of the compensating defects on our solution for the electrostatics (band bending). The actual distribution of states on the surface is not the same as assumed in Figs. 7(c) and 8, but rather, it consists of the band of intrinsic step states that is nearly filled with electrons and above which is a smaller density of states derived from the compensating defects. The Fermi level would thus lie near the top of the intrinsic band. Judging from the measured spectra, the band of extrinsic states associated with the compensating defects is 1 or 2 orders of magnitude smaller in state-density than the intrinsic states of the step edge. However, importantly, that reduction in state-density does not produce any significant change in the computed results, since even at that reduced level the state-density of the step is large enough to strongly constrain ("pin") the 
Fermi level. Therefore, our computed results remain unchanged even when the statedensity is modified to include the band of extrinsic states above the intrinsic states of the step edge.

\section{Summary}

In summary, we have studied both [001] (As-edge) and [001] (Ga-edge) steps on $\operatorname{GaAs}(110)$ surfaces with varying amounts of doping. We have focused on the As-edge steps on n-type or undoped surfaces for which the steps display no apparent reconstruction, suggestive of a simple As-terminated structure having As DBs on the step edge. These As-edge states are found to be negatively charged on n-type surfaces, as expected for transfer of electrons from the $\mathrm{CB}$ to the step edge. Tunneling spectroscopy reveals two bands of states within the band gap, one band extending out from the VB up to about $0.4 \mathrm{eV}$ above the VB maximum, and a second band having states distributed throughout the gap, at higher energies than the first band, and merging into the $\mathrm{CB}$. The former band is identified as arising from the As DBs. The latter higher energy band of states is tentatively associated with compensating defects that form along the step edge. These defects act to donate electrons to the As DBs, thereby reducing the electrostatic energy of the system.

This work was supported by the U.S. National Science Foundation, grant DMR0856240, and the Deutsche Forschungsgemeinschaft, grant Eb197/3-1. P.E. thanks C. Domke for participation in some of the experiments. 


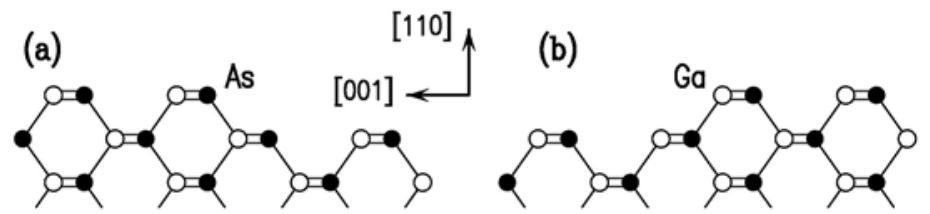

FIG 1. Schematic side view of bulk-terminated steps on the GaAs(110) surface having outward normal direction of (a) [00 $\overline{1}]$ and (b) [001]. The orientation of the former is referred to as "As-edge" and the latter as "Ga-edge". For the structures shown, the steps are terminated by As-atoms and Ga-atoms, respectively, although reconstructions of the step edges resulting in different terminations are possible.
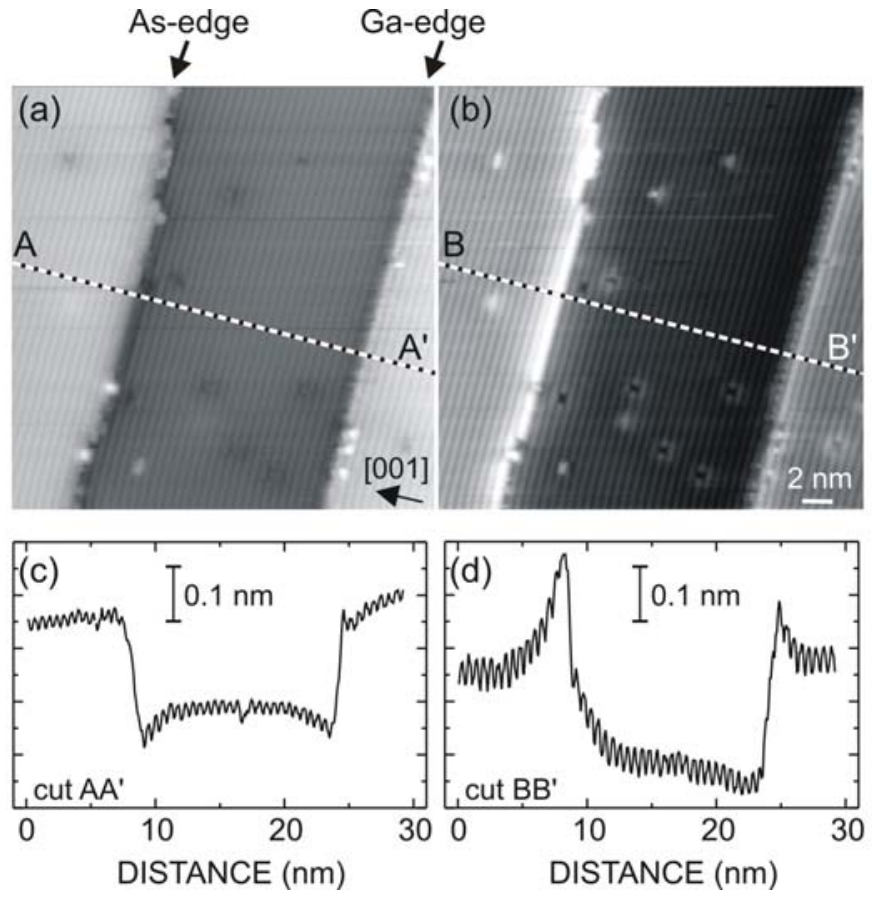

FIG 2. STM image of steps on n-type GaAs(110), acquired with constant current of 0.3 $\mathrm{nA}$ and at sample voltages of (a) +2.0 and (b) $-2.0 \mathrm{~V}$. Cuts through each image are shown below the images. 

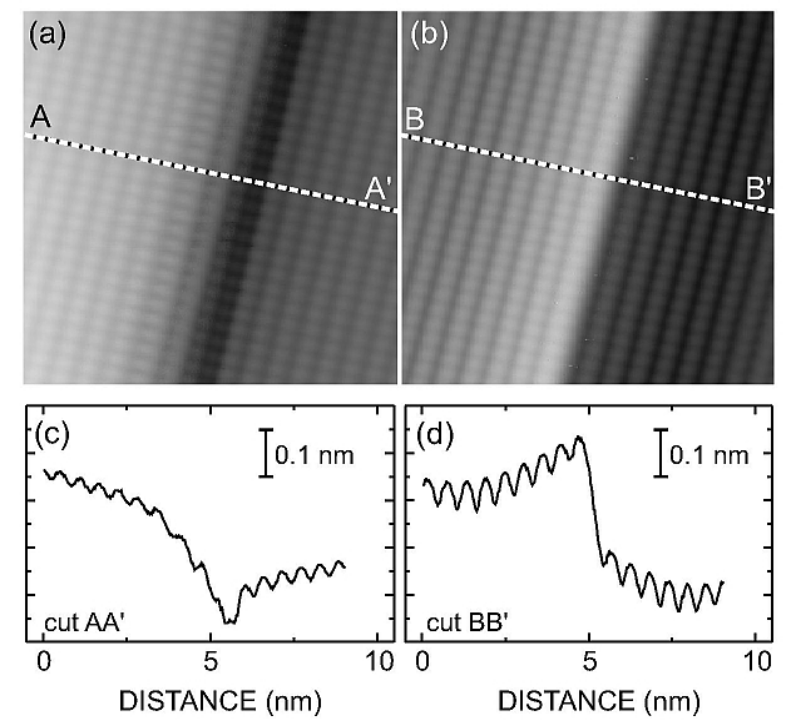

FIG 3. High resolution STM image of a step with [00 1 ] normal vector on n-type $\operatorname{GaAs}(110)$, acquired with constant current of $0.3 \mathrm{nA}$ and at sample voltages of (a) +2.0 and (b) $-2.0 \mathrm{~V}$. Cuts through each image are shown below the images.

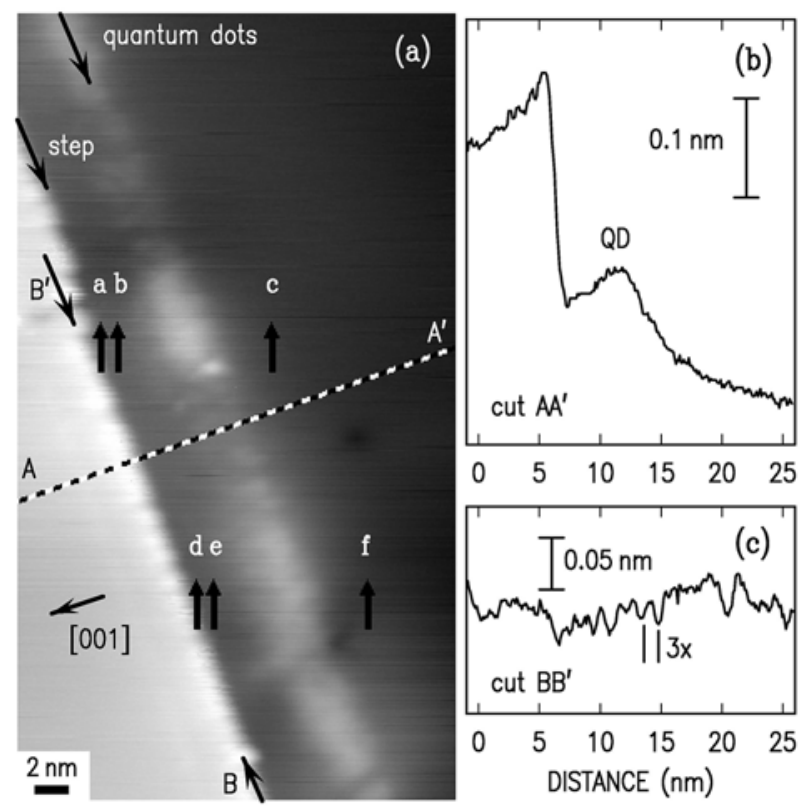

FIG 4. (a) Cross-sectional STM image of an undoped InAs/GaAs heterostructure, grown in the [001] direction, showing a layer of InAs quantum dots and a nearby step. The image was acquired with constant-current of $0.1 \mathrm{nA}$ and sample voltage of $-2.0 \mathrm{~V}$. A cross-sectional cut AA along the dashed line is shown in panel (a), and a cut between the arrows marked BB is shown in panel (c). Tunneling spectra were acquired at the locations (at the tip of the arrowhead) marked $a-f$. 


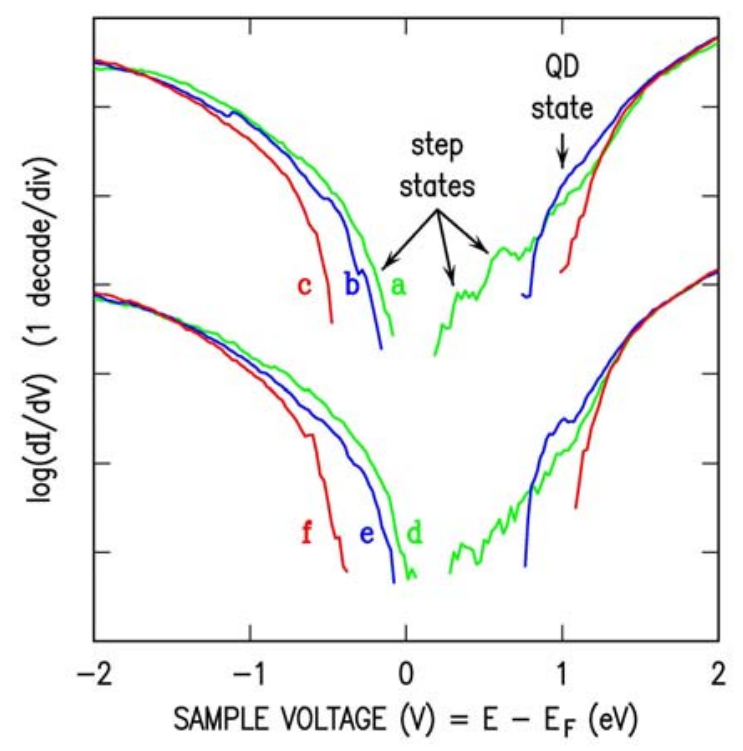

FIG 5. Tunneling spectra acquired from the surface locations near the As-edge step shown in Fig. 4(a). Spectra $a$ and $d$ were acquired at $1 \mathrm{~nm}$ from the step, $b$ and $e$ at $2 \mathrm{~nm}$ from the step, and $c$ and $f$ at $12 \mathrm{~nm}$ from the step. States induced by the step are indicated, as is a state associated with nearby quantum dots (QD).
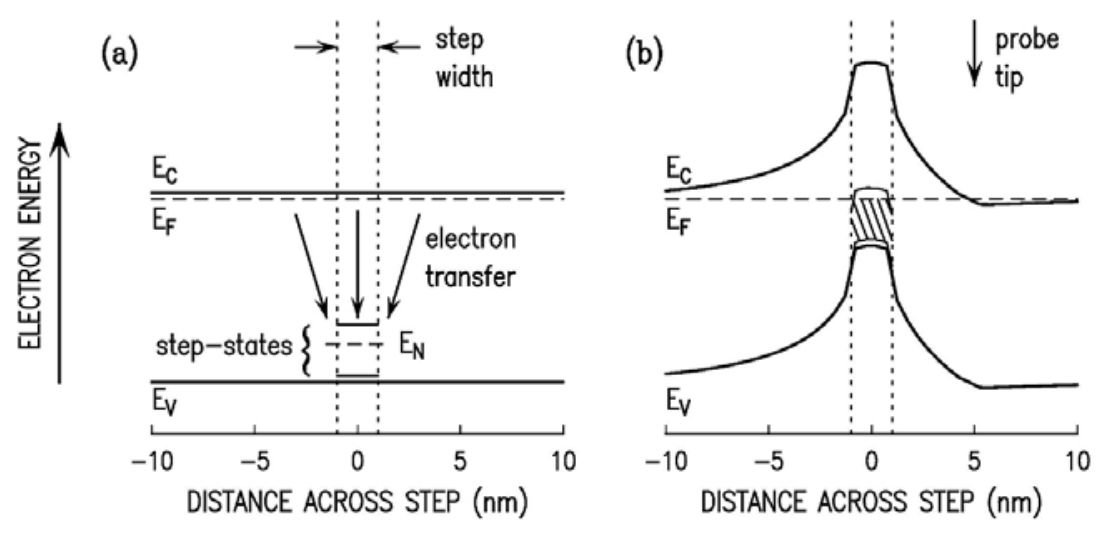

FIG 6. Band diagrams across a semiconductor surface, showing the the VB edge $\left(E_{V}\right)$ and $\mathrm{CB}$ edge $\left(\mathrm{E}_{\mathrm{C}}\right)$. A step is located at $0 \mathrm{~nm}$, with an assumed DOS of step states spread about over $2 \mathrm{~nm}$ centered on the step. $\mathrm{E}_{\mathrm{N}}$ is the charge neutrality level, above which states are negatively charged when occupied and below which they are positively charged when unoccupied. With the step states positioned lower in energy than the Fermi-level $\left(\mathrm{E}_{\mathrm{F}}\right)$ as in (a), electron transfer occurs from the CB to the step states thereby producing the band bending shown in (b). The band of step states fills up with electrons [cross-hatched region in (b)], thus producing charge on the step. The computed potential in (b) also includes the effect of a probe-tip located at $+5 \mathrm{~nm}$ from the step, with electrostatic potential energy of the probe-tip assumed to be $-2 \mathrm{eV}$ relative to a point far inside the semiconductor (i.e. a sample-tip voltage of $-2 \mathrm{~V}$ ). 

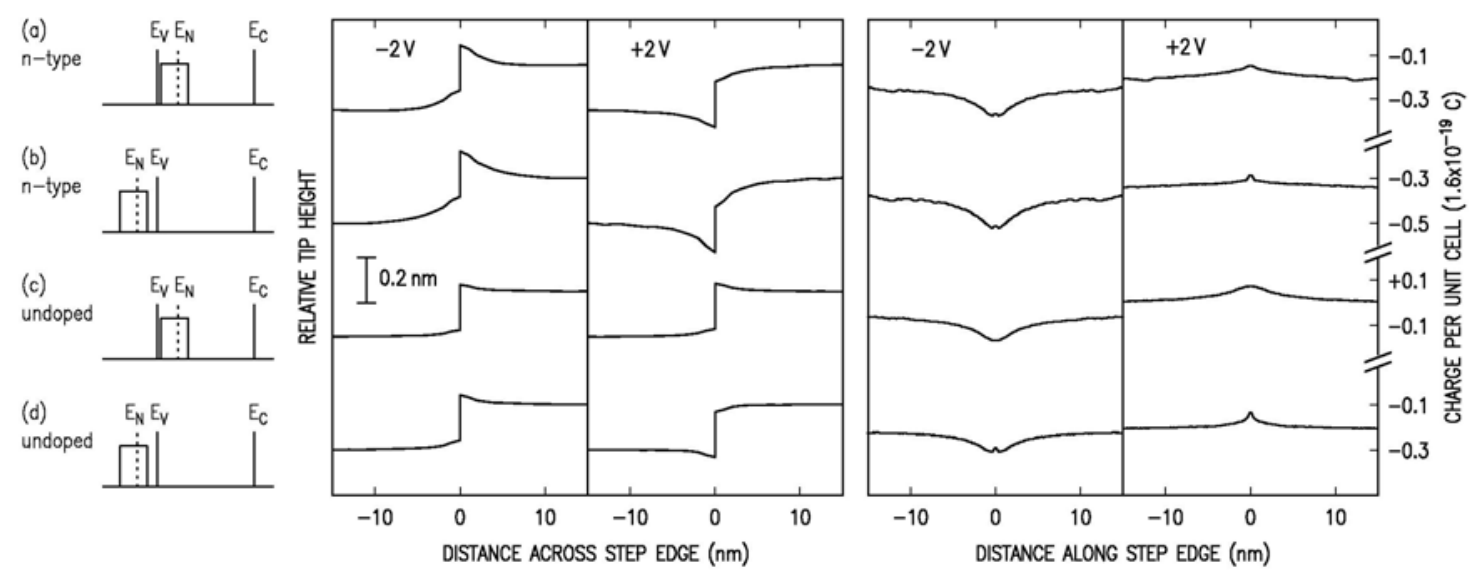

FIG 7. Computational results for four situations of doping and step state DOS, as pictured on the left-hand panels. The next two panels to the right show computed constant-current contours as the tip is scanned across the step edge. The two panels on the far right show the charge on the step edge, for a fixed tip position over the step and as a function of distance along the step edge relative to the point where the tip is located (note the different scale for the charge, on the far right-hand axis).

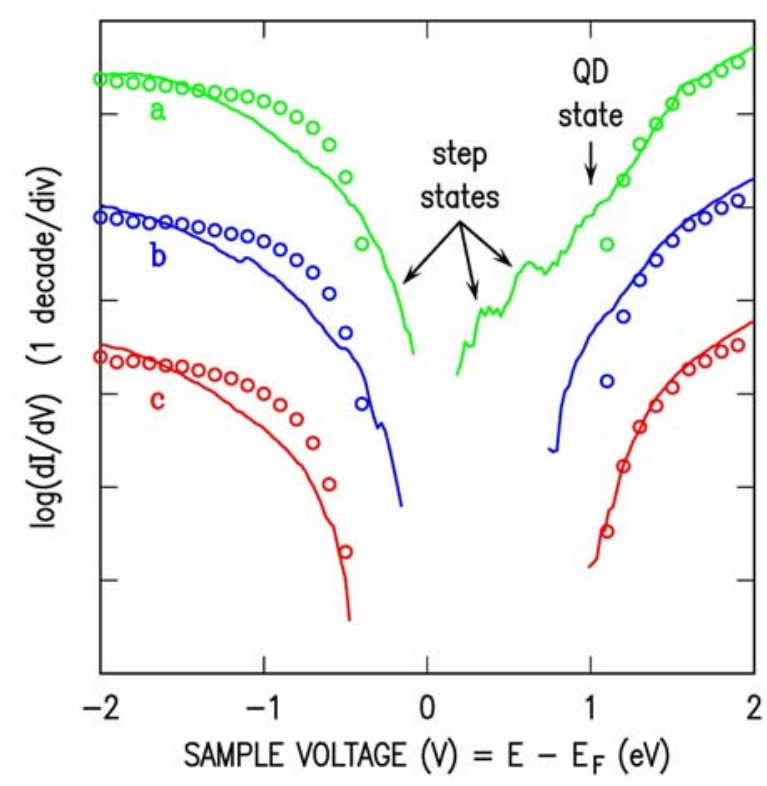

FIG 8. Comparison of measured (solid lines) and computed (open circles) spectra. The measured spectra are the same as shown in the upper part of Fig. 5, with spectrum $a$ acquired $1 \mathrm{~nm}$ from an As-edge step, $b$ at $2 \mathrm{~nm}$ from the step, and $c$ at $12 \mathrm{~nm}$ from the step. The computations use the same DOS pictured in Fig. 7(c). The computed spectra only model the GaAs bulk-like states, not the states associated with the surface steps (nor the QD state). 


\section{References}

${ }^{1}$ E.g., E. Schröer-Bergen and W. Ranke, Surf. Sci. 236, 103 (1990); R. Kaspi, and J. P. Loehr, Appl. Phys. Lett. 71, 3537 (1997); L. Masson and F. Thibadua, Phys. Rev. B 71, 85314 (2005); L. Fernandez, M. Loffler, J. Cordon, and E. Ortega, Superlattice Microst. 44, 378 (2008).

${ }^{2}$ M. Heinrich, C. Domke, Ph. Ebert, and K. Urban, Phys. Rev. B 53, 10894 (1996).

${ }^{3}$ Y. Rosenwaks, R. Shikler, Th. Glatzel, and S. Sadewasser, Phys. Rev. B 70, 085320 (2004).

${ }^{4}$ M. Kemerink, T. C. G. Reusch, D. M. Bruls, P. M. Koenraad, H. W. M. Salemink, and J. H. Wolter, Physica E 13, 1159 (2002).

${ }^{5}$ J. M. McCoy and J. P. LaFemina, Phys. Rev. B 54, 14511 (1996).

${ }^{6}$ H. Chuan Kang, X. F. Chen, and H. S. Tan, J. Chem. Phys. 107, 5914 (1997).

${ }^{7}$ J. A. Stroscio, R. M. Feenstra, and A. P. Fein, Phys. Rev. Lett. 58, 1668 (1987).

${ }^{8} \mathrm{Ph}$. Ebert, to be published.

${ }^{9}$ S. Gaan, G. He, R. M. Feenstra, J. Walker, and E. Towe, J. Appl. Phys. 108, 114315 (2010).

${ }^{10}$ R. M. Feenstra, J. Vac. Sci. Technol. B 21, 2080 (2003).

${ }^{11}$ R. M. Feenstra, Y. Dong, M. P. Semtsiv, and W. T. Masselink, Nanotechnology 18, 044015 (2007).

${ }^{12}$ Parameters for the present computations, in the notation of Ref. [8], are $s=1.0 \mathrm{~nm}, R=$ $1.5 \mathrm{~nm}, \Delta \phi=0 \mathrm{eV}$, and with values for the state-density and charge neutrality level of the step edge states specified in the text.

${ }^{13}$ N. D. Jäger, E. R. Weber, K. Urban, and Ph. Ebert, Phys. Rev. B 67, 165327 (2003).

${ }^{14}$ N. Ishida, K. Sueoka, and R. M. Feenstra, Phys. Rev. B 80, 075320 (2009).

${ }^{15}$ The increased topographic height due to charging of the step edge may appear at first glance to be much larger in the experimental results of Fig. 4(b) than in the theoretical results of Fig. 7(c). However, the experiment includes significant contributions due to the nearby quantum dot. If we examine data without the presence of a quantum dot we find a $0.06 \mathrm{~nm}$ increase at the step edge in the experiment (at $-2 \mathrm{~V}$ ), compared to 0.03 $\mathrm{nm}$ in the theory. The experiment, however, has the reduced $\kappa$ value discussed in the text by approximately a factor of 2 , consideration of which leads to reasonable resolution between the experiment and theory.

${ }^{16}$ G. Dujardin, A. J. Mayne, and F. Rose, Phys. Rev. Lett. 89, 036802 (2002).

${ }^{17}$ N. D. Jäger, E. R. Weber, K. Urban, R. Krause-Rehberg, and Ph. Ebert, Phys. Rev. B 65, 195318 (2002).

${ }^{18}$ R. M. Feenstra, S. Gaan, G. Meyer and K. H. Rieder, Phys. Rev. B 71, 125316 (2005).

${ }^{19}$ J. Mysliveček, A. Stróżecka, J. Steffl, P. Sobotík, I. Ošt'ádal, and B. Voigtländer, Phys. Rev. B 73, 161302(R) (2006).

${ }^{20}$ S. Nie, R. M. Feenstra, Y. Ke, R. P. Devaty, and W. J. Choyke, J. Appl. Phys. 103, 013709 (2008). 